EPJ Web of Conferences 53, 08011 (2013)

DOI: $10.1051 /$ epjconf/20135308011

(C) Owned by the authors, published by EDP Sciences, 2013

\title{
The Air Microwave Yield (AMY) experiment to measure the GHz emission from air shower plasmas
}

\author{
J. Alvarez-Muñiz ${ }^{1}$, M. Bohacova ${ }^{2}$, G. Cataldi ${ }^{3}$, M.R. Coluccia ${ }^{3,4}$, P. Creti $^{3}$, \\ I. De Mitri ${ }^{3,4}$, C. Di Giulio ${ }^{5}$, R. Engel ${ }^{6}$, P. Facal San Luis ${ }^{7}$, M. Iarlori ${ }^{8}$, \\ D. Martello ${ }^{3,4}$, M. Monasor ${ }^{7}$, L. Perrone ${ }^{3,4}$, S. Petrera ${ }^{8}$, P. Privitera ${ }^{7}$, \\ M. Riegel ${ }^{6}$, V. Rizi ${ }^{8}$, G. Rodriguez Fernandez ${ }^{5}$, F. Salamida ${ }^{9}$, G. Salina ${ }^{5}$, \\ M. Settimo ${ }^{10}$, R. Smida ${ }^{6}$, V. Verzi ${ }^{5, a}$, F. Werner ${ }^{6}$ and C. Williams ${ }^{7}$ \\ ${ }^{1}$ Depto. de Fisica de Particulas, Universidad de Santiago de Compostela, Santiago de \\ Compostela, Spain \\ ${ }^{2}$ Institute of Physics, Academy of Sciences of the Czech Republic, Prague, Czech Republic \\ ${ }^{3}$ Sezione INFN, Lecce, Italy \\ ${ }^{4}$ Dipartimento di Matematica e Fisica Ennio De Giorgi, Università del Salento, Lecce, Italy \\ ${ }^{5}$ Sezione INFN, Roma Tor Vergata, Italy \\ ${ }^{6}$ Forschungszentrum Karlsruhe, Institut für Kernphysik, Karlsruhe, Germany \\ ${ }^{7}$ University of Chicago, Enrico Fermi Institute Kavli Institute for Cosmological Physics, \\ Chicago, USA \\ ${ }^{8}$ Dipartimento di Fisica, Università dell'Aquila and sezione INFN, l'Aquila, Italy \\ 9 Institut de Physique Nucléaire d'Orsay (IPNO), Université Paris 11, CNRS-IN2P3, France \\ ${ }^{10}$ Universität Siegen, Germany
}

\begin{abstract}
The AMY experiment aims to measure the Microwave Bremsstrahlung Radiation (MBR) emitted by air-showers secondary electrons accelerating in collisions with neutral molecules of the atmosphere. The measurements are performed at the Beam Test Facility (BTF) of Frascati INFN National Laboratories and the final purpose is to characterize the process to be used in a next generation detectors of ultra-high energy cosmic rays (up to $10^{20} \mathrm{eV}$ ). We describe the experimental set-up and the first test measurement performed in November 2011.
\end{abstract}

\section{INTRODUCTION}

The most common experimental techniques used for the detection of ultra-high energy cosmic rays (above $10^{17}-10^{18} \mathrm{eV}$ ) are based on the detection of the fluorescence light produced during the development of the atmospheric shower and on the sampling of the secondary particles of the shower at the ground. These two techniques are employed in the Pierre Auger Observatory [1], which covers an area of $3000 \mathrm{~km}^{2}$ and consists of an array of Cherenkov detectors and a system of fluorescence telescopes. These telescopes, observing the longitudinal development of the showers, provide a calorimetric measurement of the energy and a reliable measurement of the depth of the shower maximum, which is an observable correlated with the mass composition of cosmic rays. The main limitation of these detectors is the reduced duty cycle (about 14\%) as they can only operate during

\footnotetext{
ae-mail: valerio.verzi@roma2.infn.it
}

This is an Open Access article distributed under the terms of the Creative Commons Attribution License 2.0, which permits unrestricted use, distribution, and reproduction in any medium, provided the original work is properly cited. 
clear and moonless nights. On the contrary, an array of surface detectors can operate in continuous mode with a duty cycle of about $100 \%$ but the reconstruction of the primary energy relies on predictions of high energy hadronic interaction models, leading to very large systematic uncertainties. Recently, a new detection technique based on the measurement of the microwave radiation emitted by the neutral molecules of the atmosphere has been proposed [2]. The emission mechanism, based on Molecular Bremsstrahlung, may originate radiation in the frequency range of few $\mathrm{GHz}$ from the interaction of low energy electrons in the plasma produced by air-showers. This detection technique would not suffer from the limitations of the standard detectors. In fact, the MBR is expected to be isotropic and un-polarized like the fluorescence light, providing in principle a calorimetric measurement of the primary energy. In addition, the microwave radiation is only marginally attenuated by the atmosphere and the measurement duty cycle is expected to be very close to $100 \%$. Although the MBR is a well known process in plasma physics, there are many uncertainties in the estimate of the emitted intensity (for example, coherent processes may deeply influence the emission mechanism). The authors of [2] reported the first evidence of the MBR emission from air shower plasmas. The measurements have been performed at the Stanford Linear Accelerator Center (SLAC) using a $\sim 1 \mathrm{~m}^{3}$ copper anechoic Faraday chamber. The power of the signal has been measured in the frequency band $1.5 \div 6.0 \mathrm{GHz}$ and it has been found to be proportional to the square of the beam intensity suggesting coherent emission. The energy deposit in cosmic ray induced showers can be significantly larger than the one produced at SLAC and, given the lack of knowledge on the plasma conditions, the extrapolation of the measured signal to the air showers is very uncertain. However, the authors reported promising results for the development of new detectors for CR physics above $10^{19} \mathrm{eV}$ and this has motivated the development of several prototypes (e.g. CROME [3] in Karlsruhe and AMBER, MIDAS and EASIER by the Pierre Auger Collaboration as described in [4]).

The recent results obtained by these prototypes $[5,6]$ disfavour the hyphothesis of a quadratic correlation between the emitted power and the air shower energy deposit. In addition, the measurements performed by the MAYBE experiment [7] at the Van De Graaff beam of the Argonne National Laboratory suggests that the MBR power scales linearly with the beam energy and the evidence of a lower signal with respect to the one measured at SLAC is also reported.

\section{THE EXPERIMENTAL APPARATUS}

The AMY project aims to measure the MBR absolute yield and its frequency spectrum between 1 and $20 \mathrm{GHz}$ at the Beam Test Facility (BTF) of the Frascati INFN National Laboratories (Italy), using an anechoic chamber being larger and improved with respect to the one used at SLAC in [2] and with a similar or higher beam intensity. Two 15 day measurement campaigns have been performed, the first one in November 2011 and the second one in May 2012. The first was dedicated to the measurement of the background and of the signal in different configurations of the apparatus, in order to characterize the detector response. The experimental setup used in the first test beam and the preliminary analysis of the collected data are described in details in the following sections.

\subsection{The particle source}

The Beam Test Facility (BTF) [8, 9] of the Frascati INFN National Laboratories is part of the $D A \Phi N E$ accelerator complex which is composed of a dedicated transfer line, driven by a pulsed magnet, that allows to divert electrons or positrons from the end of the high intensity LINAC toward a $100 \mathrm{~m}^{2}$ experimental hall (Fig. 1, left). The LINAC can provide electron bunches with a charge up to $10^{10} \mathrm{e}^{-}$/pulse, in an energy range between 25 and $750 \mathrm{MeV}$ with a bunch length between $1-10 \mathrm{~ns}$ and a maximum repetition rate of $50 \mathrm{~Hz}$. The bunches are accelerated by the LINAC and they are made of several microbunches with a FWHM of $14 \mathrm{ps}$ separated by the LINAC frequency of $2.856 \mathrm{GHz}$. The beam intensity is measured by an Integrating Current Transformer BERGOZ ICT 122-070-05-1 placed at the end of the beam pipe in the BTF hall. 


\section{UHECR 2012}
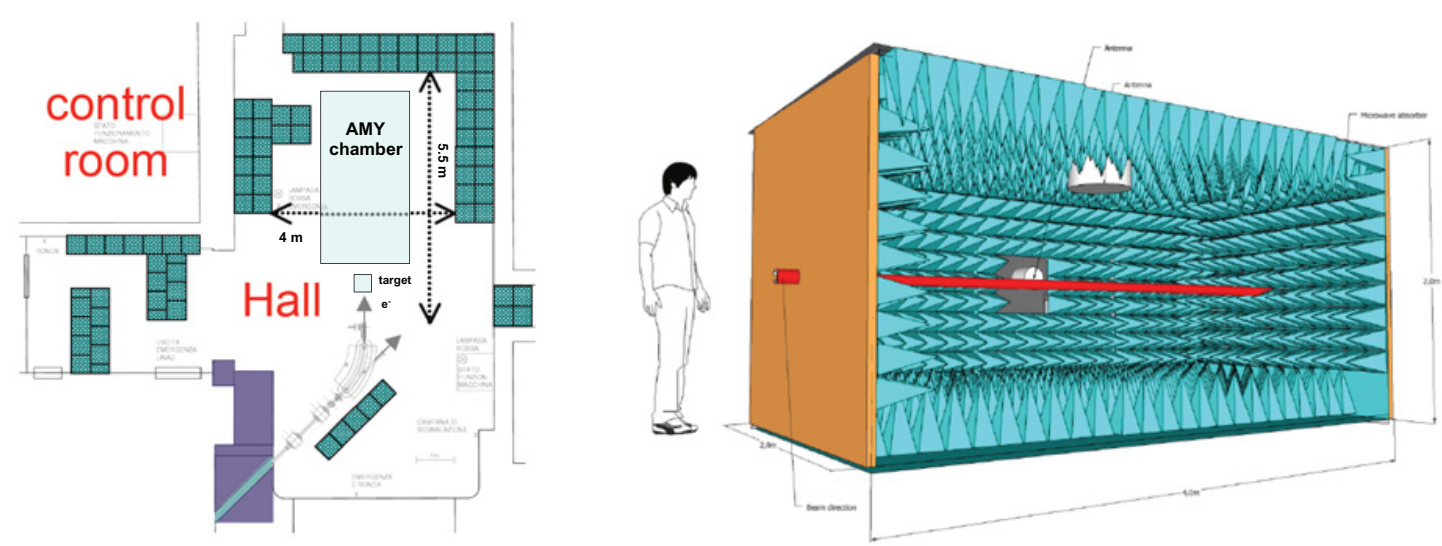

Figure 1. Layout of the BTF Hall (left) and a sketch of the AMY anechoic chamber (right).

In the first measurements campaign a $10 \mathrm{~ns}$ bunch was used with a charge up to a few $10^{9} \mathrm{e}^{-} / \mathrm{pulse}^{1}$. In order to increase the energy deposit within the chamber and therefore the sensitivity to the MBR, the beam has been collided with an alumina target, placed between the particle beam and the chamber (see next section).

\subsection{The anechoic chamber and the DAQ system}

A scheme of the copper anechoic chamber is shown in Fig. 1 (right). The inner surface is covered with pyramidal RF absorbers (AEP-12 model) which have a height of $30 \mathrm{~cm}$ and an absorption range from $35 \mathrm{~dB}$ at $1 \mathrm{GHz}$ to $45 \mathrm{~dB}$ at $6 \mathrm{GHz}$ and $50 \mathrm{~dB}$ at higher frequencies. The dimensions of the chamber $(2 \mathrm{~m}$ width $\times 4 \mathrm{~m}$ long) are mainly constrained by the space available in the BTF hall. The beam axis height is $124.5 \mathrm{~cm}$ and it crosses the chamber exactly along its central axis. Another constraint to the dimension of the chamber is set by the requirement that the distance of the antennas is large enough to guarantee the far field approximation, for which the antenna patterns are well known. For convenience the chamber has been built in 3 modules: the first and the third ones have a length of $1.5 \mathrm{~m}$ and the second is $1 \mathrm{~m}$ long. Laboratory measurements show that the shielding of the chamber from outside radiation is better than $85 \mathrm{~dB}$ above $4 \mathrm{GHz}$ and about $40 \mathrm{~dB}$ at $1 \mathrm{GHz}$. A picture of the anechoic chamber installed in the BTF hall during the first measurement campaign is shown in Fig. 2 on the left.

The energy deposit within the chamber was increased by colliding the beam particles with a target consisting of $95 \% \mathrm{Al}_{2} \mathrm{O}_{3}$ and placed between the beam and the chamber. During the first campaign a few test runs were acquired with the a target of $20 \mathrm{~cm}$ thickness placed at $50 \mathrm{~cm}$ from the chamber. This corresponds to about $\approx 2.7 X_{0}$ (radiation lengths) and it is slightly above the maximum shower development which occurs at $\approx 1.3 X_{0}$.

One of the aims of the experiment is to measure the MBR dependence on the energy deposited by the shower particles. For this purpose an compressed air system allowing a remote control of the target thickness has been built. The system allows the selection of 6 different configurations with a total thickness ranging from $2.5 \mathrm{~cm}$ up to $45 \mathrm{~cm}$. This system has been used for the first time in the test of May 2012.

As shown in Fig. 2 (left), the AMY chamber may host up to 5 different radio receivers in the positions A, B (looking at the beam from behind), C, D (at the side of the beam) and T (from the top). The

\footnotetext{
${ }^{1}$ Measurements with a bunch length of $3 \mathrm{~ns}$ and with a higher intensity have been performed in May 2012.
} 

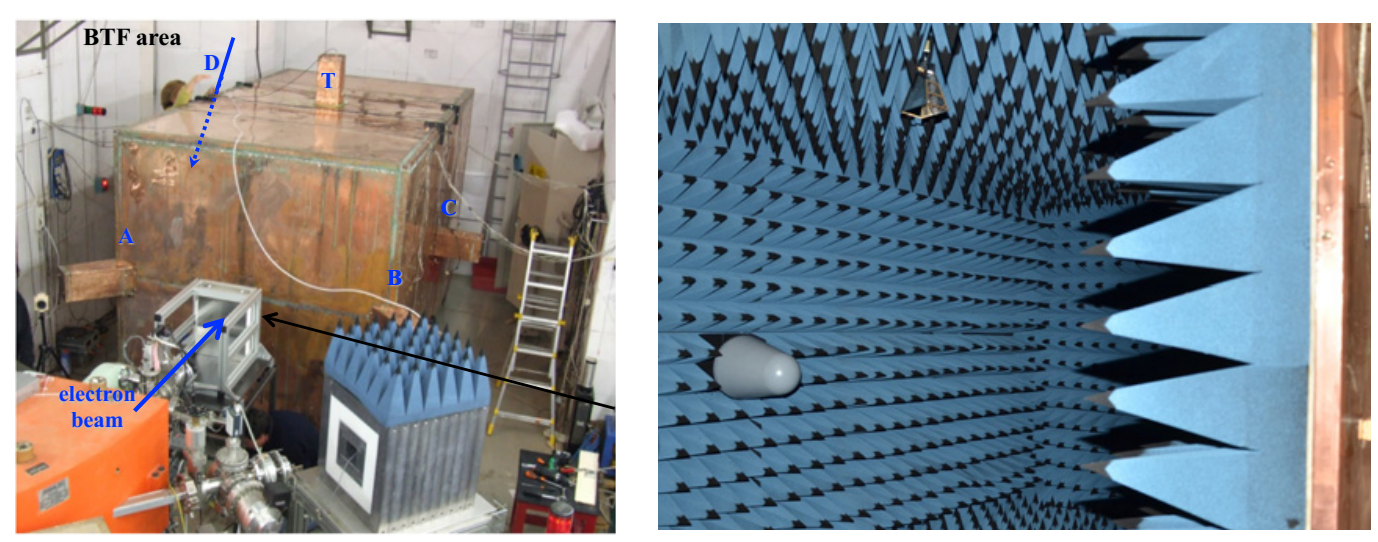

Figure 2. Left: picture of the AMY anechoic chamber in the BTF hall during the first measurements campaign in November 2011. The antennas can be installed in 5 different positions: 2 at the corner (A and B), 2 at the side of the chamber (C and D) and one looking from the top (T). The direction of the beam is also shown while the black arrow indicates the alumina target. Right: interior part of the chamber with two antennas installed. The antenna on the top is the horn while the one on the side is the log-periodic.

support of the radio receivers allow us to change the distance of the antennas from the beam axis and to rotate their polarization plane. Two orientations were used, with the polarization plane of the antennas orthogonal (cross-polarized) and parallel (co-polarized) to the beam axis. The main motivations for using these configurations is discussed in the next section.

The experimental setup includes two Rohde\&Schwarz $(R \& S) H L 050$ log-periodic antennas and two RF Spin Double Ridged Waveguide Horn DRH2O. They are shown on the right picture of Figure 2. Both the horn and the log-periodic antennas have a broadband input frequency $(1.7-20 \mathrm{GHz}$ and $0.85-20 \mathrm{GHz}$ respectively). The log-periodic antennas have the advantage of a quite regular radiation patterns over the full frequency range but they are sensitive to the high noise level in the low frequency range $(<1 \mathrm{GHz})$ where the chamber does not attenuate properly the external radiation. This problem has been overcome by applying software and/or hardware filters. The gain of the two receivers is at level of $12 \mathrm{dBi}$ for the log-periodic antennas and from $6 \mathrm{dBi}$ up to $16 \mathrm{dBi}$ for the horn.

The output signal of each radio receiver was amplified by about $26 \mathrm{~dB}$ by the Minicircuits wide band amplifier ZVA-183-S+ $(800 \mathrm{MHz}-21 \mathrm{GHz})$. The signals have been sent through $\approx 20 \mathrm{~m}$ low loss cables to the control room and acquired by a Lecroy SDA 830Zi-A oscilloscope. It has 4 input channels with a $20 \mathrm{GHz}$ real time bandwidth and a sampling rate of $40 \mathrm{GS} / \mathrm{s}$. The channels have been calibrated using a Rohde\&Schwarz SMF100A signal generator (range $100 \mathrm{kHz}-22 \mathrm{GHz}$ ) and a Rohde\&Schwarz SFSV30 spectrum analyzer (range $9 \mathrm{kHz}-30 \mathrm{GHz}$ and $40 \mathrm{MHz}$ bandwidth). The power loss in the cables becomes significant at the higher frequencies. The overall calibration curve (amplifier + cables) decrease approximately in a linear way from $20 \mathrm{~dB}$ to about $-10 \mathrm{~dB}$ in the frequency range of interest.

The antenna signals were acquired in a $500 \mathrm{~ns}$ time window in correspondence of the presence of the beam in the BTF. The signal trigger has been extracted from the DAFNE timing system, allowing a very precise (few ps) time decision. One channel of the oscilloscope has been used to monitor the beam intensity inspecting the signal of the Integrating Current Transformer available in the BTF. This allowed us to measure the beam intensity for each particle bunch crossing the chamber.

\section{DATA ANALYSIS}

Figure 3 shows an example of the raw traces acquired by the oscilloscope. The picture on the left shows the Integrating Current Transformer signal (trace in green) for one bunch of particles. The number of 

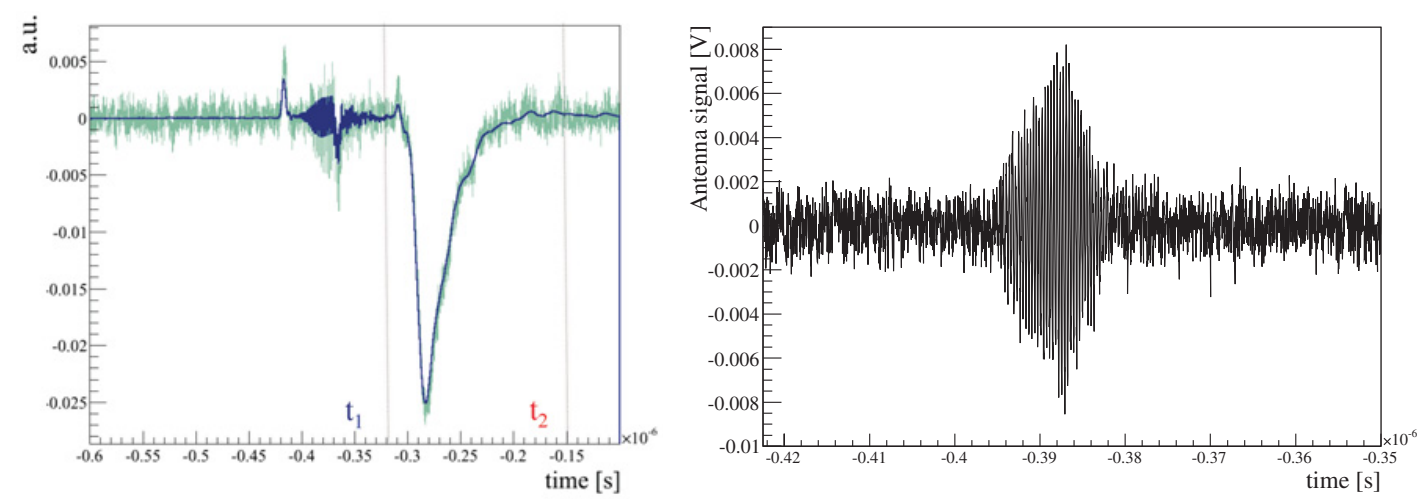

Figure 3. Left: oscilloscope trace of the Integrating Current Transformer signal used to measure the beam intensity. The green line refers to one bunch of particles, while the blue line refers to the signal averaged over many triggers. In order to emphasize the high time precision of the trigger signal, both curves have been normalized to same number of particles and have been plotted after the subtraction of the pedestal. Right: oscilloscope trace of the horn signal.

beam particles is estimated by making the integral in a time window centered on the peak and subtracting the pedestal estimated in the first part of the track. The calibration factor to obtain the beam intensity is provided by the BTF staff. In order to prove the high time precision of the trigger, the beam signal for one bunch is compared with the beam signal averaged over many triggers (the curves have been normalized and the pedestal subtracted).

The picture on the right shows the trace of the antenna as observed on the oscilloscope. A clear signal is seen and the root mean square is calculated to estimate the corresponding power $\left(P=V_{r m s}^{2} / 50 \Omega\right)$. This trace has been detected for one trigger with high beam current. For triggers with less particles the signal is smaller and the noise detected by the antenna due to the amplifier and the oscilloscope is not negligible. This background noise has been estimated by making the root mean square of the first part of the track, i.e. when the beam has not yet entered the chamber. The background estimate was checked in several ways, by taking data with the LINAC being ON but without electron beam in the BTF line and with electrons in the chamber but obscuring the antenna field of view with RF absorbers. In all cases the background has been found compatible to the one derived from the first part of the trace. This technique is highly preferred as it allows us a background estimate for each signal.

One of the key points of the measurement is the understanding of the radiation produced directly by the beam. Even if the alumina target decreases the average energy of the particles, most of them produce Cherenkov radiation which is not negligible in the microwave band. This radiation is clearly a background process for the MBR measurement and, in order to minimize it, we adopted the approach suggested in [2]. The Cherenkov radiation has a well defined polarization, lying in the plane defined by the Poynting vector and the particle trajectory. Therefore the Cherenkov contribution can be minimized by orienting the polarization plane of the antenna orthogonal to the beam axis ${ }^{2}$. Our data confirm that the cross-polarized signal is significantly smaller than the co-polarized one. The ratio of the two signals measured with the horn is at the level of $15 \div 20 \mathrm{~dB}$.

Figure 4 left, shows the correlation between the power detected by the horn and the number of electrons per bunch. The antenna was cross-polarized and placed at the center of the chamber (position C). No filters have been applied and therefore the effective bandwidth is close to $20 \mathrm{GHz}$. The signal is clearly well correlated to the beam, showing a quadratic scaling and therefore suggesting coherent

\footnotetext{
2 The MBR radiation is not polarized and then its measurement is not sensitive to the particular orientation of the antenna polarization plane.
} 

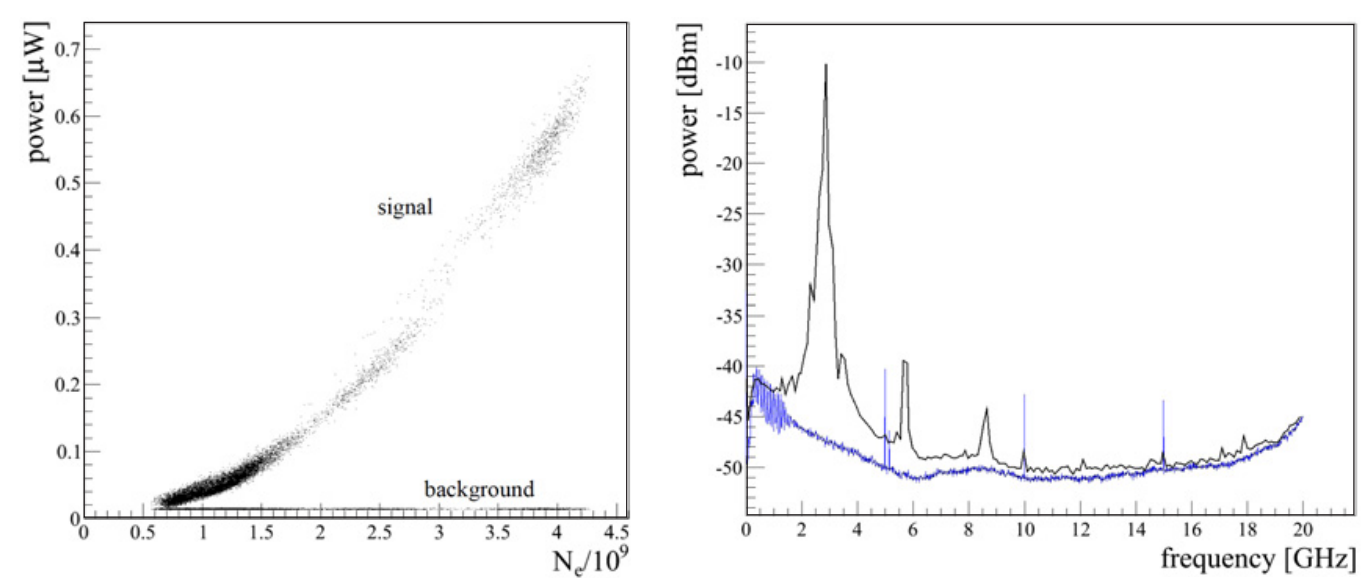

Figure 4. Left: correlation between the power of the signal and the beam intensity given in number of electrons per bunch and normalized to $10^{9}$ particles $\left(N_{e} / 10^{9}\right)$. The background contribution is shown together with the signal. Right: frequency spectrum of the signal (black) and of the background(blue) obtained by making a Fourier transform of the oscilloscope trace. The spectrum has been obtained averaging over many triggers, giving a significant reduction of the statistical fluctuations.

emission. As shown in Figure 4 left, the background noise is mostly independent of the beam intensity and then negligible at the highest intentities.

Figure 4 right, shows the frequency spectrum of the signal (black) and of the background (blue). They have been obtained using the Fourier Transform of the oscilloscope traces and averaging over many triggers. The spectrum is dominated by peaks centered at multiples of the LINAC frequency $\left(f_{L}=2.856 \mathrm{GHz}\right)$. A preliminary simulation of the Cherenkov radiation qualitatively reproduces the observed spectrum. The peaks are due to the constructive interference of the radiation emitted by the beam microbunches and the power of the signal scales quadratically with the beam intensity.

Even if the simulation gives some hints to explain the data, we can not draw any conclusion without a measurement of the Cherenkov contribution. For example we can not exclude that the MBR spectrum would have the same structure with the same kind of peaks. Moreover, comparing the spectrum of the signal with the one of the noise (see Fig. 4, right), we notice that there is some power outside the peaks and it would be interesting to understand if this power is compatible or not with the Cherenkov process. The detailed simulation of the Cherenkov signal and of the detector, currently underway, together with the analysis of the data collected in May, will allow us the understanding of the physical process and its detection.

\section{CONCLUSIONS}

We have presented the AMY experiment conceived to measure Microwave Bremsstrahlung Radiation emitted by secondary electrons accelerated in collisions with neutral molecules of the atmosphere. We have described the experimental setup and the preliminary analysis of 2011 data. Data have shown that the key point of the measurement is the estimate of the radiation directly produced by the beam particles. A detailed simulation of the Cherenkov process and the detector response is underway and soon we will present the analysis of the data collected in May 2012. 


\section{UHECR 2012}

We warmly acknowledge B. Buonomo, L. Foggetta, G. Mazzitelli and all the Frascati Accelerator Division technical staff for their continuous cooperation and support. This work is partly supported by the INFN and by the European Community through the TARI project (N. 29). The BTF and its staff are partly supported by the AIDA Grant Agreement no. 262025.

\section{References}

[1] The Pierre Auger Collaboration [J. Abraham et al.], Nucl. Instrum. Meth. A 523, (2004) 50

[2] P.W. Gorham et al., Phys. Rev. D 78 (2008), 032007

[3] R. Smida et al., Results from the CROME experiment, Proceeding of the 31st Int. Cosm. Ray Conf. (ICRC 2011), arXiv:1108.0588

[4] The Pierre Auger Observatory, Proceedings of the 31st Int. Cosm. Ray Conf. (ICRC 2011), arXiv:1107.4807

[5] P. Facal et al., These proceedings

[6] J. Alvarez-Muñiz et al., submitted to PRL, arXiv:1205.5785v1

[7] C. Williams et al., These proceedings

[8] B. Buonomo, G. Mazzitelli, P. Valente, IEEE Trans. Nucl. Sci. 52 (2005) 824

[9] B. Buonomo, G. Mazzitelli, F. Murtas, L. Quinter, Proceeding of BIW08, Tahoe City, California 